\title{
Comparative study of hydrogen and methane flames under oxygen enrichment
}

\author{
Swati Bhardwaj* and Pramod Bhatia \\ IITM University, Gurgaon
}

\section{(C)2016 ACCENTS}

\begin{abstract}
Computational studies of methane and hydrogen laminar jet inverse diffusion flames have been discussed herein. The flame configurations are modelled using computational tool UNICORN. Flame length, width and adiabatic temperature for methane and hydrogen with increasing oxygen content have been reported in the literature and results of both hydrogen and methane are compared. The salient feature in the combustion phenomenon of these fuels is that the flame length, width and temperature are invariably proportional to oxygen enrichment.
\end{abstract}

\section{Keywords}

Hydrogen, Methane, Oxygen enrichment, Laminar flames, IDF.

\section{Introduction}

Unlike normal flames, where the fuel is surrounded by oxidizer, inverse flames are those where oxidizer is surrounded by fuel. Past work revealed some of the characteristics for inverse diffusion flames. Quenching limits of laminar axisymmetric inverse diffusion flames were measured by Zhang Yi [1]. The flames were obtained by injecting various oxidizers into fuels. Takagi T. [2] numerically investigated preferential diffusion effects on the temperature in normal and inverse $\mathrm{H} 2 / \mathrm{N} 2$ diffusion flames. The process of accumulation or dilution of $\mathrm{H} 2$ species and excess and deficit of enthalpy were analysed and effect of flame curvature were also analysed. Lecoustre V. [3] et. al. numerically investigated quenching limits of hydrogen/air spherical diffusion flames for normal and inverse configurations. The burners were adiabatic porous spheres of various sizes. The flames were simulated using a steady-state laminar flame code with detailed chemistry and transport. It was found that for equivalent heat release rates, inverse flames are smaller and hotter than normal flames. Miao J [4] investigated circumferential-fuel-jets inverse diffusion flame (CIDF) burner burning liquefied petroleum gas (LPG) enriched with $50 \%$ of hydrogen fuel (H2) equivalence ratio and fuel jet velocity of LPG of the $50 \% \mathrm{H} 2-\mathrm{LPG}$ mixed fuel was identified.

*Author for correspondence
Experiments were also carried out to investigate the flame structures of the LPG flame and LPG enriched $\mathrm{H} 2$ flame were experimentally investigated and both flames were compared in addition to this flame height at various equivalence ratio were also investigated. Bindar Y. [5] experimentally investigated the size and structure of LPG and Hydrogen inverse diffusion flames. Lim K. [6] experimentally investigated the spontaneous ignition of a hydrogen jet emanating from a slot into an oxidizing ambient (e.g., air).

\section{Computational study}

In the present study species of fuel are Hydrogen (H2) and Methane (CH4) with the following computational test conditions given in Table 1.

Table 1 Computational study

\begin{tabular}{ll}
\hline Fuels & $\begin{array}{l}\text { Hydrogen }\left(\mathbf{H}_{2}\right), \text { Methane } \\
\left(\mathbf{C h}_{4}\right)\end{array}$ \\
\hline Oxygen by volume in N2 & $21 \%, 30 \%, 50 \%$ and $100 \%$ \\
Gravity & Earth gravity $(1-\mathrm{g})$ \\
Fuel velocity & $0.866 \mathrm{~m} / \mathrm{s}$ \\
Air velocity & $1 / 10$ th of fuel velocity \\
& $(0.0866 \mathrm{~m} / \mathrm{s})$ \\
\hline
\end{tabular}

Computational tool used to study flame behaviour of laminar flames: A time-accurate CFDC code known as UNICORN is used to predict flame behaviour of Methane and Hydrogen flames under varying oxygen concentration. UNICORN is a timedependent, axisymmetric mathematical model that solves for axial- and radial-momentum equations, 
continuity, and enthalpy- and species-conservation equations to simulate a variety of dynamic jet flames. The governing equations, written in the cylindricalcoordinate system, are as follows:

$$
\begin{aligned}
& \frac{\partial(\rho)}{\partial t}+\frac{1}{r} \frac{\partial(r \rho u)}{\partial r}+\frac{\partial(\rho v)}{\partial z}=0 \\
& \frac{\partial(\rho \Phi)}{\partial t}+\frac{\partial(\rho u \Phi)}{\partial r}+\frac{\partial(\rho v \Phi)}{\partial z}=\frac{\partial}{\partial r}\left(\Gamma^{\phi} \frac{\partial \Phi}{\partial r}\right)+\frac{\partial}{\partial z}\left(\Gamma^{\phi} \frac{\partial \Phi}{\partial z}\right)-\frac{\rho u \Phi}{r}+\frac{\Gamma^{\phi}}{r} \frac{\partial \Phi}{\partial r}+S^{\Phi}
\end{aligned}
$$

\section{Grid and domain optimization}

The present simulations involved a round $5.5 \mathrm{~mm}$ diameter burner and quiescent ambient gas at 0.98 bar and 298 K. Figure 1 presents the geometry of the axisymmetric computational domain. The computational domain used is $100 \mathrm{~mm}$ and $50 \mathrm{~mm}$ in the axial ( $\mathrm{z}$ ) and radial (r) directions, respectively, and domain is represented by a $201 \times 101$ grid system. The co-flow velocity is set at $1 / 10$ th of the jet velocity. The effect of the round $5.5 \mathrm{~mm}$ diameter burner on flow dynamics is modelled by including an adiabatic rectangular body of thickness $0.45 \mathrm{~mm}$ inside the computational domain. The body height is $10 \mathrm{~mm}$.

Computational Results for Methane: Figures (1), (2), (3) and (4) represent the temperature profile of methane diffusion flames under oxygen enrichment.

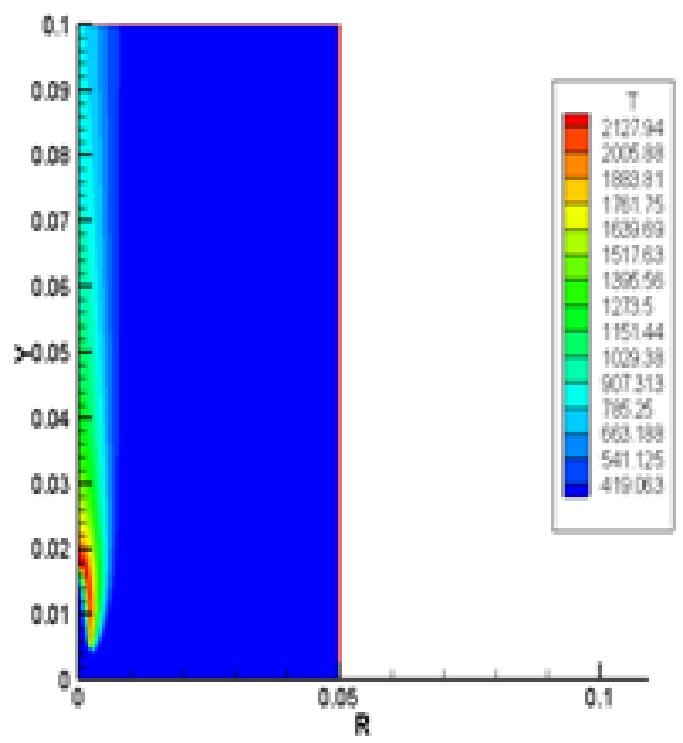

Figure 1 Temperature profile of methane in $21 \%$ oxygen

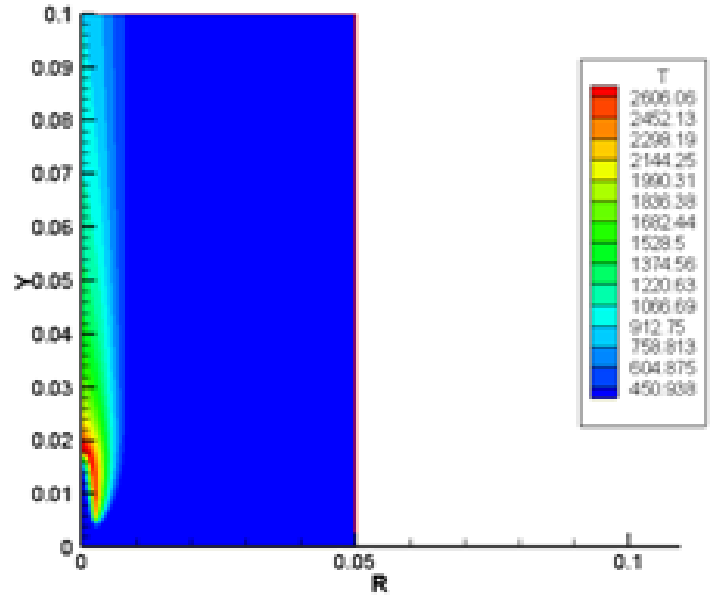

Figure 2 Temperature profile of methane in $30 \%$ oxygen

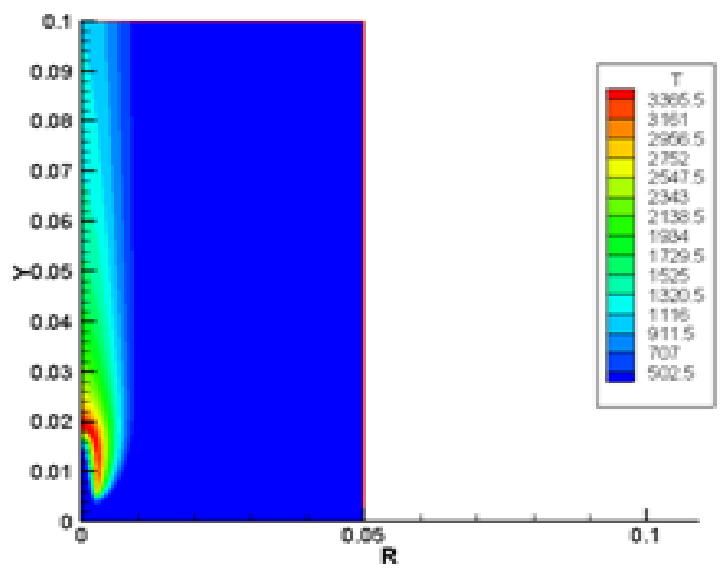

Figure 3 Temperature profile of methane in $50 \%$ oxygen

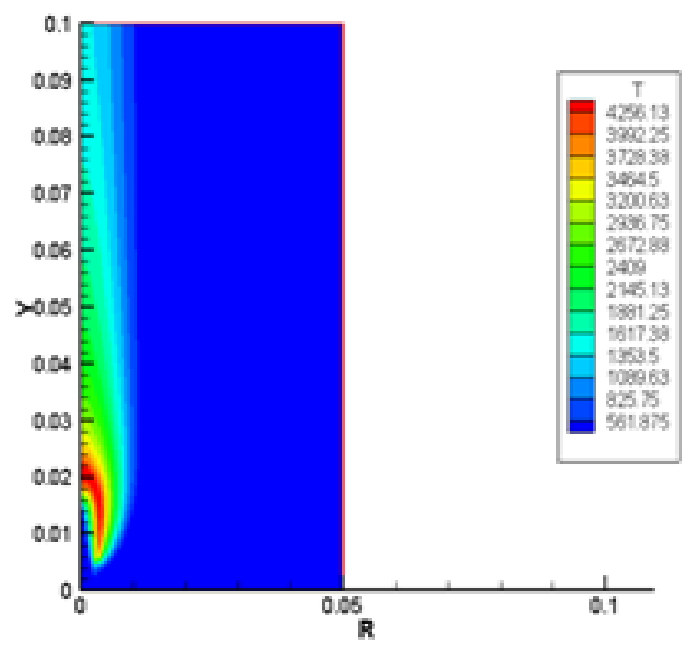

Figure 4 Temperature profile of methane in $100 \%$ oxygen 
Swati Bhardwaj et al.

Figures (5), (6), (7) \& (8) represent the temperature profile of hydrogen diffusion flames under oxygen enrichment.

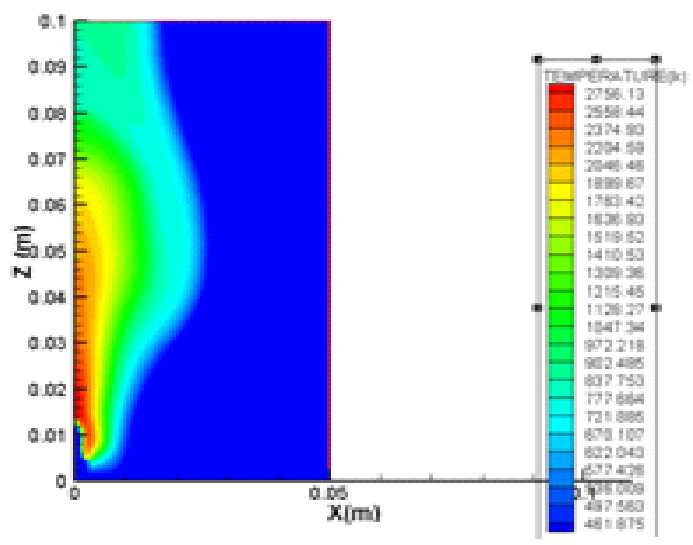

Figure 5 Temperature profile of hydrogen in $21 \%$ oxygen

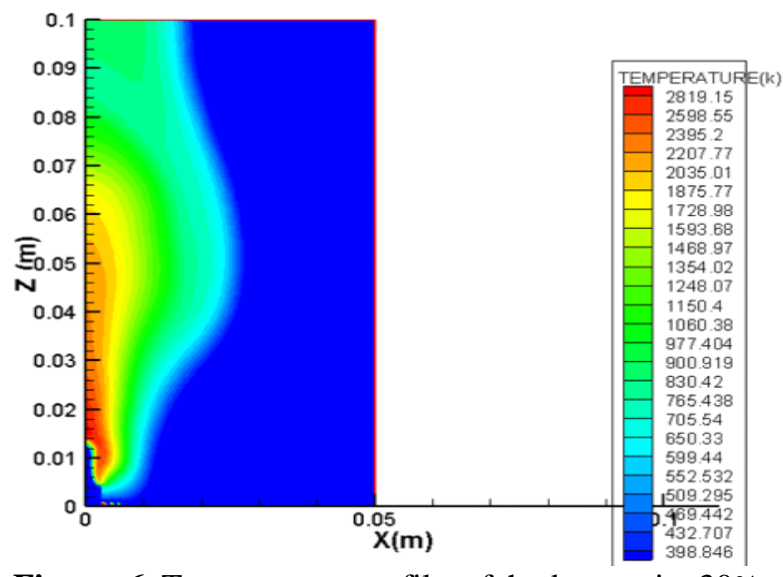

Figure 6 Temperature profile of hydrogen in $30 \%$ oxygen

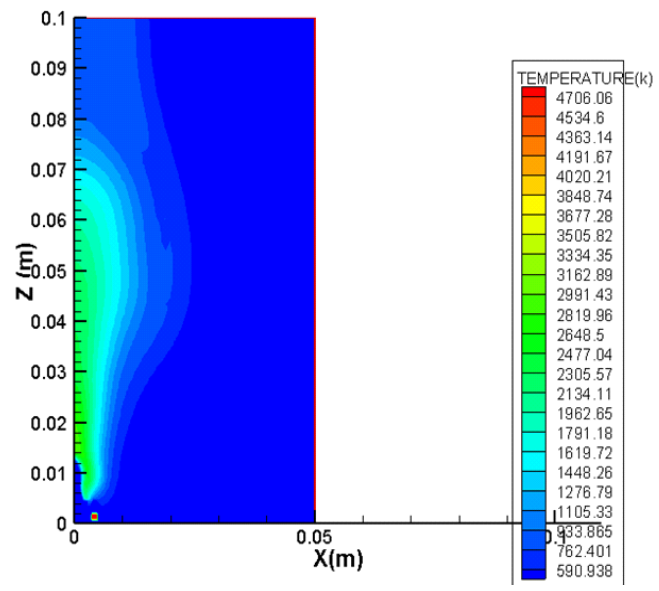

Figure 7 Temperature profile of hydrogen in $50 \%$ oxygen

60

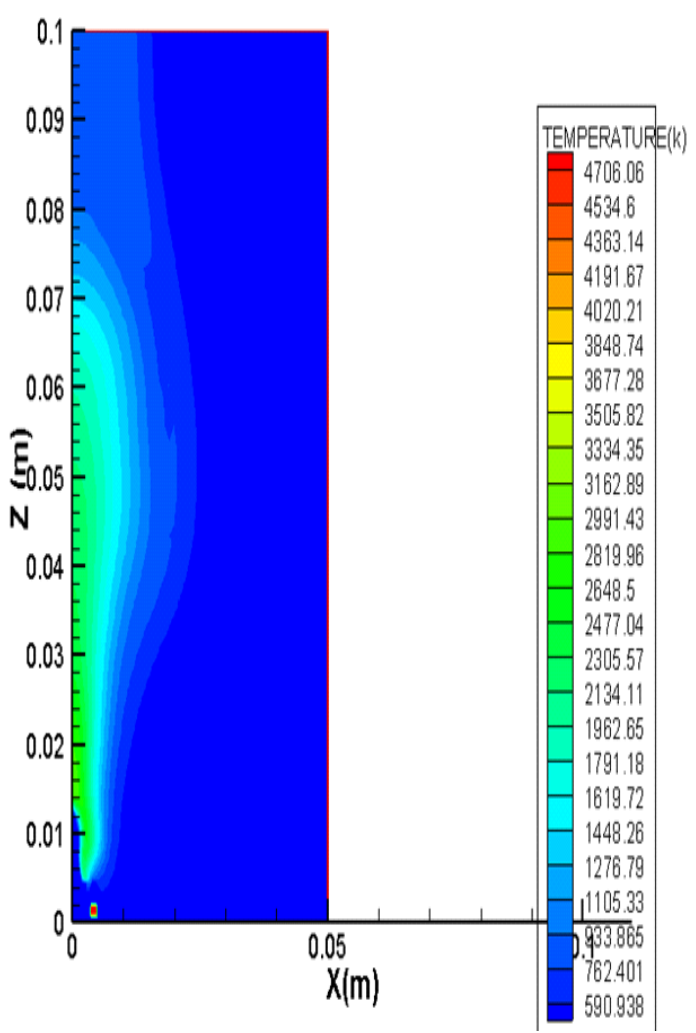

Figure 8 Temperature profile of hydrogen in $100 \%$ oxygen

Flame length: Flame length of IDF of methane and hydrogen is shown in Figures [1-8] with increasing oxygen content. In inverse diffusion flames length of flame increases slightly oxygen enhancement.

Flame width: Flame width of IDF of methane and hydrogen is shown in Figures [1-8] with increasing oxygen content. In inverse diffusion flames width of flame increases slightly with gravity reduction and oxygen enhancement.

\section{Adiabatic flame temperature}

Adiabatic flame temperature of IDF of methane and hydrogen is shown in Figs [1-8] with increasing oxygen content. In inverse diffusion flames width of flame increases slightly with gravity reduction and oxygen enhancement.

\section{Conclusion}

Flame luminosity and visible flame length of diffusion flames of methane and hydrogen increase with increase of oxygen concentration. Soot production increases with oxygen concentration in case of methane and there is no soot production in case of hydrogen because absence of carbon content. 
The salient feature in the combustion phenomenon of these fuels is that the flame length, width and temperature are invariably proportional to oxygen enrichment. Adiabatic flame temperature in flame increases with increase of oxygen concentration in both the cases. It is found that adiabatic flame temperature of hydrogen is more than methane. In case of methane height of flame is more than hydrogen flame.

\section{Future work}

As has already been expressed, the present works primary focus has been on laminar diffusion flames, however, almost all the practical combustion applications involve turbulence. In future turbulence modelling can be done by researchers.

\section{Acknowledgment}

None.

\section{Conflicts of interest}

The authors have no conflicts of interest to declare.

\section{References}

[1] Zhang Y., Peter, Sunderland P., Measurement of Inverse diffusion flame quenching limits (Doctoral dissertation). Spring Technical Meeting of the Central States Section of the Combustion Institute. 2012; 224.

[2] Takagi T, Xu Z, Komiyama M. Preferential diffusion effects on the temperature in usual and inverse diffusion flames. Combustion and Flame. 1996; 106(3):252-60.

[3] Lecoustre VR, Sunderland PB, Chao BH, Axelbaum RL. Modeled quenching limits of spherical hydrogen diffusion flames. Proceedings of the Combustion Institute. 2013; 34(1):887-94.

[4] Miao J, Leung CW, Cheung CS. Effect of hydrogen percentage and air jet Reynolds number on fuel lean flame stability of LPG-fired inverse diffusion flame with hydrogen enrichment. International Journal of Hydrogen Energy. 2014; 39(1):602-9.

[5] Bindar Y, Irawan A. Characteristics of flame shapes and map for LPG and hydrogen inverse confined diffusion flames at high level of fuel excess. ASEAN Journal of Chemical Engineering. 2012: 12(1); 1-10.

[6] Lim KB, Chao BH, Sunderland PB, Axelbaum RL. A theoretical study of spontaneous ignition of fuel jets in an oxidizing ambient with emphasis on hydrogen jets. Combustion Theory and Modelling. 2008; 12(6):117996. 